\title{
Lithium and proton-capture elements in globular clusters: the case of 47 Tucanae
}

\author{
Valentina D'Orazi ${ }^{1}$, Sara Lucatello ${ }^{1,2}$, Raffaele Gratton ${ }^{1}$, \\ Angela Bragaglia ${ }^{3}$, and Eugenio Carretta ${ }^{3}$ \\ ${ }^{1}$ INAF-Osservatorio Astronomico di Padova, vicolo dell'Osservatorio 5, I-35122, Padova \\ email: valentina.dorazi@oapd.inaf.it, sara.lucatello@oapd.inaf.it, \\ raffaele.gratton@oapd.inaf .it \\ ${ }^{2}$ Excellence Cluster Universe, Boltzmannstr. 2, D-85748, Garching, Germany \\ ${ }^{3}$ INAF-Osservatorio Astronomico di Bologna, via Ranzani 1, I-40127, Bologna \\ email: angela.bragaglia@oabo.inaf.it; eugenio.carretta@oabo.inaf.it
}

\begin{abstract}
The determination of lithium (Li) abundances in Globular Clusters (GCs), along with proton-capture elements $(\mathrm{Na}, \mathrm{O}, \mathrm{Mg}, \mathrm{Al})$, offer a key tool to address the pollution scenario and its mechanisms, the dilution process acting within each star and the first phases in the lifetime of GCs. We present our results on $\mathrm{Na}, \mathrm{O}$ and $\mathrm{Li}$ abundance determination in a large sample of dwarf stars in the GC 47 Tucanae (NGC 104). While we found a clear Na-O anti-correlation, in perfect agreement with giant members by Carretta et al. (2009a,b), Li abundance appears neither positively correlated with oxygen, nor anti-correlated with sodium. Our finding unveils an intrinsic scatter in $\mathrm{Li}$ content, independent of intra-cluster pollution by a first generation of more massive, faster evolving stars.
\end{abstract}

Keywords. Stars: abundances - Galaxy: globular clusters: individual: 47 Tuc

\section{Introduction}

The traditional assumption of GCs as simple stellar populations has been proven too simplistic. In the last years a lot of observational studies revealed the presence of anticorrelations between $\mathrm{C}, \mathrm{O}, \mathrm{Mg}$ and $\mathrm{N}, \mathrm{Na}, \mathrm{Al}$, respectively, pointing out to self-enrichment processes within GCs, and to the existence of multiple stellar generations. In this context, $\mathrm{Li}$ abundances can provide very powerful tracers of cluster formation and evolution, because this element is easily destroyed in the stellar interiors. Regardless of the nature of the polluters (AGB stars -Ventura \& D'Antona 2009- or fast rotating massive stars -Decressin et al. 2007), the Li content in polluting matter must be very close to zero (although in some cases AGB stars can also produce Li). The determination of Li variations in GCs allows us to uniquely constrain the nature and the extent of the dilution process between pristine and polluted material. Specifically two main issues can be addressed: (1) Do entirely polluted ( $\mathrm{Li} \simeq 0$ ) stars exist? (2) Is the minimum detectable Li the same in all GCs or, otherwise, can it significantly vary from cluster-to-cluster? The latter point has also strong implications on the GC formation and early evolution. Here we present our results on the metal-rich $([\mathrm{Fe} / \mathrm{H}]=-0.76$, Carretta et al. 2009c) GC 47 Tuc.

\section{Sample and analysis}

We retrieved from the ESO Archive (Programme 081.D-0287, PI P. Shen) FLAMESGIRAFFE spectra of 109 unevolved, turnoff (TO) member stars of 47 Tuc. Gratings HR15n, HR18 and HR20a were employed to cover the Li I (6707 ̊̊), O I (7771, 7773, 

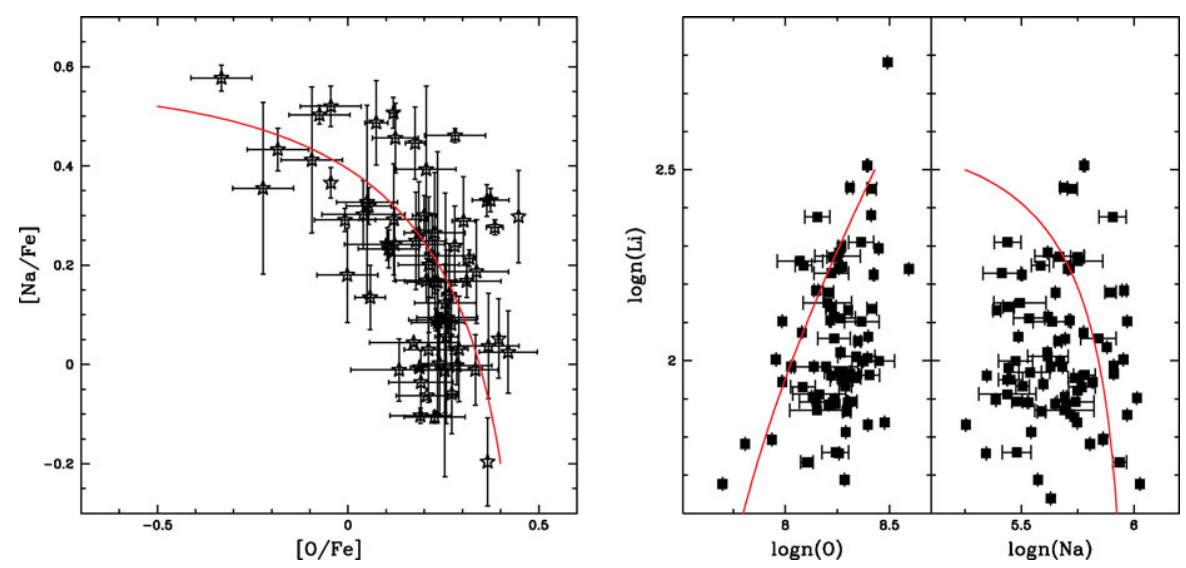

Figure 1. Na-O anticorrelation (left panel) and Li-Na-O distributions in dwarf stars of 47 Tuc (right panels).

$7774 \AA)$, and Na I $(8183,8194 \AA)$ features, respectively. The analysis was carried out using the ROSA code (developed by R. Gratton) and Kurucz model atmospheres; for Na and $\mathrm{O}$, we derived abundances from the equivalent widths, applying corrections due to NLTE effects; LTE Li abundances were instead obtained through spectral synthesis.

\section{Results and discussion}

As shown in Fig. 1, the unevolved cluster stars reveal a very clear Na-O anti-correlation, confirming what has been obtained for giant members (Carretta et al. 2009a). The Na-O anti-correlation is well described by a simple dilution model (red solid line in Fig. 1), like the one proposed by Prantzos \& Charbonnel (2006). On the other hand, Li abundances show a wide scatter, in both the Li-O and Li-Na planes, with values often well below those expected from a simple dilution model. Furthermore, the cluster behaviour seems different from that of NGC 6397 (Lind et al. 2009), a GC much more metal-poor than 47 Tuc $([\mathrm{Fe} / \mathrm{H}]=-1.99$, Carretta et al. 2009c) and for which Lind et al. detected a significant Li-Na anti-correlation. Note that turnoff stars in 47 Tuc are cooler $\left(\mathrm{T}_{\text {eff }} \sim 5700-5800\right.$ $\mathrm{K})$ than those in NGC $6397\left(\mathrm{~T}_{\text {eff }} \sim 6100-6300 \mathrm{~K}\right)$, being well below the limit usually considered for the Spite plateau. Finally, the large scatter observed in 47 Tuc seems reminiscent of the wide $\mathrm{Li}$ variations observed in the old open cluster M 67 (Randich et al. 2000) and, in general, in old thick disk stars with $\mathrm{T}_{\text {eff }} \approx 5800 \mathrm{~K}$ (Ryan et al. 2001): this is the first time that this phenomenon is observed in GC stars.

\section{References}

Carretta, E., Bragaglia, A., Gratton, R., et al. 2009a, A\&GA, 505, 117

Carretta, E., Bragaglia, A., Gratton, R., \& Lucatello, S. 2009b, A\& A, 505, 139

Carretta, E., Bragaglia, A., Gratton, R., D’Orazi, V., \& Lucatello, S. 2009c, A\& $A, 508,695$

Decressin, T., Charbonnel C., Prantzos, N. \& Ekstrom, S. 2007, A\& A, 464, 1029

Lind, K., Primas, F., Charbonnel, C., Grundahl, F., \& Asplund, M. 2009, A\&AA, 503, 545

Prantzos, N. \& Charbonnel, C. 2006, A\&A, 458, 135

Randich, S., Pasquini, L., \& Pallavicini, R. 2000, A\& $A$, 356, 25

Ryan, S., Kajino, T., \& Beers, T. 2001, ApJ, 549, 55

Ventura, P. \& D'Antona, F. 2009, A\&A, 499, 835 